\title{
AN OPTICAL FLOW-BASED SENSING SYSTEM FOR REACTIVE MOBILE ROBOT NAVIGATION
}

\author{
Eliete Maria de Oliveira Caldeira* \\ eliete@ele.ufes.br
}

\author{
Hans Jörg Andreas Schneebeli* \\ hans@ele.ufes.br
}

\author{
Mário Sarcinelli-Filho* \\ mario.sarcinelli@ele.ufes.br
}

* Departamento de Engenharia Elétrica - Universidade Federal do Espírito Santo

Av. Fernando Ferrari, 514, 29075-910 Vitória/ES Brazil

Voice: +55 2740092684 Fax: +55 2740092737

\begin{abstract}
This work discusses the use of optical flow to generate the sensorial information a mobile robot needs to react to the presence of obstacles when navigating in a non-structured environment. A sensing system based on optical flow and time-to-collision calculation is here proposed and experimented, which accomplishes two important paradigms. The first one is that all computations are performed onboard the robot, in spite of the limited computational capability available. The second one is that the algorithms for optical flow and time-to-collision calculations are fast enough to give the mobile robot the capability of reacting to any environmental change in real-time. Results of real experiments in which the sensing system here proposed is used as the only source of sensorial data to guide a mobile robot to avoid obstacles while wandering around are presented, and the analysis of such results allows validating the proposed sensing system.
\end{abstract}

KEYWORDS: Optical flow; Mobile robots; Autonomous vehicles; Obstacle avoidance; Mobile robot navigation; Computer vision.

\footnotetext{
Artigo submetido em 10/01/2006

1a. Revisão em 19/01/2007

2a. Revisão em 19/04/2007

Aceito sob recomendação do Editor Associado

Prof. José Reinaldo Silva
}

\section{INTRODUCTION}

Many applications of mobile robots demand the use of a CCD camera fixed onboard the mobile platform, like surveillance, remote inspection, etc. Thus, most mobile platforms include such sensor as part of its hardware resources.

In addition, vision is very likely to be the richest human sensing subsystem, for the wide gamma of information it can provide (Farah, 2000; Ramel, 2000). Then, to make profit of the vision system available onboard the mobile robot is quite reasonable. However, for the sake of cost reduction, the vision system frequently available onboard the robot is a monocular one, thus imposing severe limitations on how to use vision to get useful information about the surrounding environment. Information about the amount of objects in the scene, for example, could be generated by simply segmenting an acquired image (Tekalp, 1995; Adams and Bischof, 1994; Bleau and Leon, 2000), but the relative depth of such objects would not be determined, thus making impossible to the robot to implement the necessary maneuvers to avoid them.

Techniques exist, however, that allow the mobile robot to navigate without colliding to any obstacle. One of the most well known among such techniques is the map of potentials, which is a collection of range measurements defining preferential directions of movement so that the robot reaches its goal and avoids obstacles (Barraquand, Langlois and Latombe, 1992). The key variable to determine such vectors is the distance from the robot to an obstacle (Barraquand, 
Langlois and Latombe, 1992), which can be measured using sonar or laser range finder.

Regarding monocular vision, a technique exists that is able to provide information about the distance from a camera fixed onboard the robot to an object, which is based on the optical flow vector field (Horn and Schunck, 1981). The optical flow field is obtained by processing two or more sequential views of the same scene. Such technique has been explored in many applications, like plant growth monitoring (Barron and Liptay, 1997), video compression (Wallach, Kunapalli and Cohen, 1994), automatic car driving (Giachetti, Campani and Torre, 1998; Batavia, Pomerlau and Thorpe, 1998), and mobile robot navigation (Ancona and Pogio, 1995; Carelli et. al., 2002; Dev, Kröse and Groen, 1997a; Baratoff, Toepfer and Neumann, 2000; Camus et. al., 1999; Coombs et. al., 1998; Santos-Victor et. al., 1995; Stöfler, Burkert and Färber, 2000).

This paper addresses an application in mobile robot navigation that uses the optical flow technique to get all the information the robot needs to safely navigate. In some applications regarding the use of optical flow in mobile robot navigation, a special camera arrangement (Coombs et. al., 1998; SantosVictor et. al., 1995), a single special camera (Camus et. al., 1999; Coombs et. al., 1998) or specialized hardware pieces (Ancona and Pogio, 1995; Stöfler, Burkert and Färber, 2000; Carelli et. al., 2002; Dev, Kröse and Groen, 1997a; Coombs et. al., 1998) has been adopted in order to get more accurate information or to accelerate the optical flow calculation. Such resources are not available here: the only sensor used is a perspective camera with small aperture angle (48.8 degrees in the horizontal direction) fixed on the robot. Therefore, the solution adopted is to pay special attention to the calculation of the optical flow vectors.

In other applications related to mobile platforms navigation, for the restrictions on the computational capability, most results so far published consider some simplification: processing just small parts of the acquired images (Carelli et. al., 2002; Dev, Kröse and Groen, 1997a; Giachetti, Campani and Torre, 1998; Batavia, Pomerlau and Thorpe, 1998), acquiring and processing smaller images (Lorigo et. al., 1997), or even performing the calculations off-board the robot (Carelli et. al., 2002; Dev, Kröse and Groen, 1997a), are strategies commonly adopted. In the last case, a more efficient computer off-board the robot receives the images it acquires, performs the calculations and sends a control signal back to it. However, in such a case the robot autonomy is restricted, once the robot is constrained to keep close the off-board computer, for not loosing communication. Other strategy that has been recently adopted is the pre-processing of the images acquired to generate a more compact set of input data, which would cause the processing time to be reduced. However, the re- sults so far obtained have not allowed increasing the image acquisition rate (Baratoff, Toepfer and Neumann, 2000). No simplifications like those above mentioned are adopted here: the images used are 240x320 pixels grey level bitmaps, and all the calculations are performed onboard the mobile platform.

The optical flow-based sensing system here proposed generates a one-dimensional map of depths corresponding to the horizon in front of the robot, covering its entire field of vision. The behavior wandering (Brooks, 1986), meaning that the robot moves itself around just avoiding obstacles, is implemented, in order to demonstrate the capability of the optical flow-based sensing system proposed to deal with obstacle detection and avoidance. Moreover, no other sensing apparatus than the $\mathrm{CCD}$ camera is used, once the optical flow technique is able to provide the necessary sensorial information. Finally, the entire system is implemented in a computer onboard the mobile platform, thus guaranteeing its full autonomy.

The tests with the system here developed were performed with the ActivMedia Pioneer 2-DX mobile robot (see Figure 1), whose onboard computer is based on the Intel Pentium MMX $233 \mathrm{MHz}$ processor, and includes 128 Mbytes of RAM memory. The CCD camera onboard the robot is a SONY D30/D31 analog one, attached to a PC Imagenation frame grabber. The robot also embeds Saphira, an API that allows the programmer to get sensorial information and to actuate on the robot motors, through passing setup values for the heading angle and the linear speed. Actually, Saphira delivers to the robot embedded microcontroller (a Siemens $20 \mathrm{MHz} 88 \mathrm{C} 166$ one) the low-level actuation signals, in accordance with the high level commands it receives. It also allows the programmer to get information from the robot odometry, which is here used to recover the trajectory the robot followed.

For describing the system implemented, the paper is here-

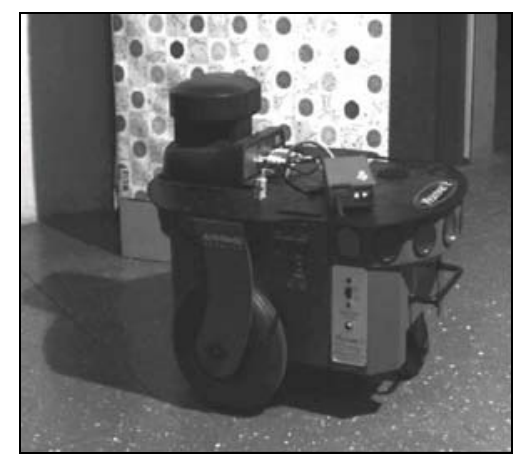

Figure 1: A photograph of the robot used in the experiments. 
inafter structured in five sections. Section 2 discusses the algorithm adopted to perform the optical flow calculation, which is one of the main responsible for guaranteeing the real-time characteristic of the whole system. In the sequence, Section 3 discusses an algorithm adopted to perform the motion segmentation (Tekalp, 1995), based on the optical flow vectors calculated, which produces the map of depths used by the robot to avoid obstacles, here referred to as the vector of times to collision or times to contact. Next, Section 4 deals with a state-machine implemented to allow the robot to avoid obstacles in some different contexts, which is based on the vector of times to collision generated in Section 3. Finally, Section 5 shows some experimental results, while Section 6 highlights the main conclusions.

\section{OPTICAL FLOW CALCULATION}

The main paradigm to be considered here is to implement an algorithm that calculates the optical flow vectors as fast as possible, in order to preserve the capability of the system of reacting to any environmental change in real time. As images should be acquired in an acquisition rate of thirty frames per second, the acquisition of several image frames would demand a lot of time, so that the first step of choosing a suitable algorithm was to impose that no more than two image frames should be acquired. Also, only gradient-based methods for optical flow calculation (Barron, Fleet and Beauchemin, 1993) were considered. Another strong limitation was not to consider iterative algorithms, like the classical one due to Horn and Schunck (1981), for the large computation time demanded to get a good optical flow vectors estimate.

Then, starting from the analysis of the methods for optical flow calculation presented in (Barron, Fleet and Beauchemin, 1993), the least-squares algorithm proposed by Lucas and Kanade (1981) was selected as the candidate to estimate the optical flow vectors. In such an algorithm, the optical flow is considered constant in a region of $\mathrm{N}$ by $\mathrm{N}$ pixels. Thus, writing the optical flow constraint for each pixel in the region one gets the set of observations

$$
I_{x_{p}} u+I_{y_{p}} v+I_{t_{p}}=0 \text { for } p=1, \ldots, N \times N,
$$

from which the least squares estimate

$$
(\hat{u}, \hat{v})=\left(\mathbf{A}^{T} \mathbf{A}\right)^{-1} \mathbf{A}^{T} \mathbf{b}
$$

of the optical flow vector is obtained, where

$$
\mathbf{A}=\left[\begin{array}{c}
I_{x_{1}}, I_{x_{2}}, \ldots, I_{x_{N \times N}} \\
I_{y_{1}}, I_{y_{2}}, \ldots, I_{y_{N \times N}}
\end{array}\right]^{T}
$$

and

$$
\mathbf{b}=-\left[I_{t_{1}}, I_{t_{2}}, \ldots, I_{t_{N \times N}}\right]^{T}
$$

Other algorithms available in the more recent literature were also tested, like those proposed in (Grossmann and SantosVictor, 1997), (Nesi, Bimbo and Ben-Tzvi, 1995) and (Lai and Vemuri, 1998), mainly for the robust and high-quality estimate they generate.

Tests run with this group of algorithms are reported in (Sarcinelli-Filho et. al., 2002b), and the main result is that the more robust algorithms demand a computation time that is much higher than that demanded to get the least-squares estimate. Hence, as low computation time is a main paradigm in the application here considered, the least squares optical flow vectors estimate was selected as the most suitable one for onboard implementation. However, to reduce the number of pixels of each one of the two image frames used when writing the observation equations, like it is done in (Grossmann and Santos-Victor, 1997), showed to be very interesting. Following this strategy, our implementation adopts regions of $10 \times 10$ pixels $(24 \times 32$ regions in each image frame) and adopts 12 observations for processing the optical flow estimate associated to each region. The computation time associated to such a modified least squares estimate is summarized in Table I. The main result of such an implementation is that real-time performance is assured to the sensing system here proposed.

However, experiments have shown that such an algorithm generates rougher estimates of the optical flow vectors, in comparison with the classical least-squares estimate. Actually, in all the experiments run it generated an underestimated time to contact when the robot approached an obstacle. Nevertheless, the rougher estimate of the optical flow vectors, from which the time to contact emerges (Dev, Kröse and Groen, 1997a), does not represent any danger to the robot, in this case, once any evasive maneuver is started in advance.

\section{OPTICAL FLOW-BASED IMAGE SEG- MENTATION}

With the objective of making easier to get information about the environment surrounding the robot, it was implemented an image segmentation step entirely based on the optical flow vectors previously calculated. The result of such segmentation is to disclose the distinct objects present in the scene.

The optical flow-based image segmentation is also called segmentation of movement, which consists in grouping the image pixels that perform the same movement. Thus, distinct groups of pixels emerging from the segmentation are associ- 
ated to different moving objects in the 3D scene (Borshukov et. al., 1997; Tekalp, 1995). Therefore, the optical flowbased segmentation corresponds to group pixels whose optical flow vectors are similar, thus corresponding to the same movement in the image frame considered. Then, considering that the optical flow field is a good representation of the field of movement, each group of pixels emerging from the segmentation is associated to a single 3D structure. An important aspect, in this sense, is that the accuracy of the result of the segmentation is straightforwardly connected to the accuracy of the optical flow vectors previously calculated (Stöfler, Burkert and Färber, 2000). Actually, after calculating the optical flow vectors as described in Section 2, each vector represents a region of $10 \times 10$ pixels in the image, such that a matrix of $24 \times 32$ different optical flow vectors is the data to be analyzed in order to detect objects in the image frame. Therefore, the image segmentation process is not time-consuming.

Table 1: Computation time associated to the least squares estimates of the optical flow.

\begin{tabular}{|c|c|}
\hline Least squares estimate & $\begin{array}{c}\text { Normalized Computation } \\
\text { time }(\%)\end{array}$ \\
\hline Classical one & 100 \\
\hline Modified one & 6.25 \\
\hline
\end{tabular}

The methods available in the literature for optical flow-based image segmentation can be grouped in three main categories (Borshukov et. al., 1997). The first one corresponds to the use of affine clustering (Wang and Adelson, 1994), meaning to cluster pixels based on an affine optical flow model. For the second category, the segmentation is based on the dominant movement (Bergen, Burt and Hanna, 1992), where in the current iteration the region of dominant movement is identified and the movement it performs is estimated. The procedure is repeated in the next iteration, not including regions previously identified as regions of dominant movement. The third category executes simultaneously the estimation and the segmentation of movements.

The method proposed by Wang and Adelson (1994) is an example of the use of affine clustering to segment movements. It supposes that the optical flow corresponding to an image frame can be described as a set of planar regions in the space of velocity, what means that the optical flow has an affine model in such regions. This way, the optical flow is estimated for the entire image, which is divided in rectangular regions, where the parameters of the affine model of the optical flow are estimated. As the models of regions corresponding to the same object in the real world, they are grouped in a small number of classes by using an adaptive k-means algorithm. An affine model is obtained for each group of regions with similar models, and then each optical flow vector is grouped in one of the resulting classes.

The multistage affine segmentation method proposed by Borshukov et al. (1997) combines the method of segmentation based on the dominant movement of Bergen et al. (1992) and the clustering using the affine model adopted by Wang and Adelson (1994). The image is divided in rectangular blocks for which the parameters of the affine model of the optical flow are estimated. The parameters associated to the dominant movement are then determined, by combining the parameters of different regions. Then, the regions whose parameters are well represented by this model are identified and grouped together. The procedure is repeated for the regions not included in any group, thus generating another vector characterizing a dominant movement, and so on.

The procedure proposed in this work uses a constant value as the model of the optical flow in each region of 10x10 pixels, in consonance with the algorithm adopted for optical flow calculation discussed in Section 2. It groups the contiguous regions characterized by optical flow vectors that are similar, according to the metric used in (Borshukov et. al., 1997) and (Wang and Adelson, 1994). Therefore, the optical flow vectors corresponding to the regions $r_{i j}$ and $r_{k l}$ are similar if

$$
\left(u_{k l}-u_{i j}\right)^{2}+\left(v_{k l}-v_{i j}\right)^{2}<T_{i j},
$$

where $i$ and $j$ indicate the row and the column of the region $r_{i j}$ under consideration in the matrix of optical flow vectors, respectively, $k$ and $l$ indicate, respectively, the row and the column of one of the regions in the neighborhood of $r_{i j}, u$ and $v$ are the components of the optical flow in the directions $x$ and $y$, respectively, and $T_{i j}$ is a threshold value for the region $r_{i j}$. Such condition corresponds to say that two neighbor regions are similar if the squared magnitude of the difference between their optical flow vectors is below a certain threshold.

Choosing the value of $T_{i j}$ is a very important issue, for a very low value could cause regions corresponding to a single $3 \mathrm{D}$ object not to be grouped during the segmentation procedure, as well as a very high value could cause the grouping of regions corresponding to different $3 \mathrm{D}$ objects. Besides, as the robot is most time approaching objects in the scene, the optical flow is expected to be a radial field of vectors. Moreover, it is expected that the magnitude of the vectors diminish when the region under consideration is close to the focus of expansion of such field. Hence, it is not possible to use a single value for $T_{i j}$ for all regions. After several experiments, it was decided to adopt $20 \%$ of the squared quadratic norm of the optical flow vector corresponding to the region $r_{i j}$ for the threshold $T_{i j}$, which presented good results.

The algorithm adopted for performing the optical flow-based 


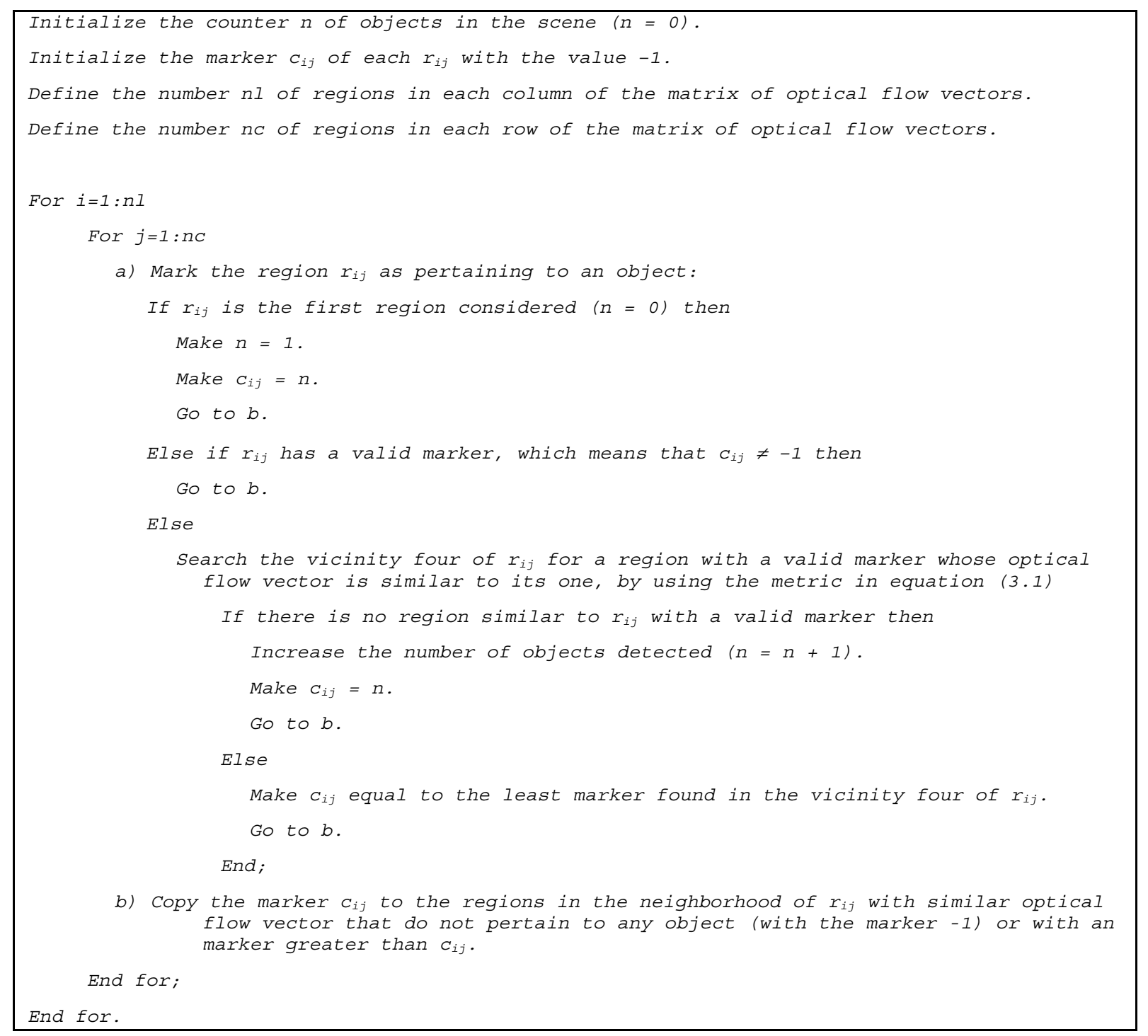

Figure 2: The optical flow-based image segmentation algorithm adopted.

image segmentation is presented in Figure 2. There, a certain region in the image is identified as $r_{i j}$, where $i$ is the row and $j$ is the column of the matrix of optical flow vectors, and the corresponding optical flow vector is characterized as $(u, v)_{i j}$. A marker $c_{i j}$ is associated to it (positive nonzero integers), which defines an object such a region is part of.

The regions for which the optical flow vectors are not available (those in which Equation (2) has no solution) are disregarded when running the algorithm of Figure 2, and their markers remain with the value -1 . As a result of running such an algorithm, each distinct marker represents a different object in the image frame considered, while the regions with a -1 marker are regions detected as not pertaining to any object. All the procedure is performed in a single sweep, thus being extremely fast, once it is run over the matrix of 24x32 optical flow vectors (not over each pixel of the 240x320 pixels image frame). Such characteristic is a very important one, when regarding that the robot should react to the presence of obstacles in real-time.

In the sequence, the focus of expansion (FOE) corresponding to the entire image frame is calculated, based on the set of valid optical flow vectors available (Dev, Kröse and Groen, 1997a). It is the point from which all optical flow vectors emerge, and both components of the optical flow vector are null in such a point $(u=0$ and $v=0)$. It is determined from the optical flow field calculated, searching for the point in which the directions of the vectors in the field cross each other (Dev, Kröse and Groen, 1997a). Having the coordi- 
nates of the FOE and the optical flow field, one can calculate the time to contact correspondent to each region of the image under consideration. It is performed through the equation

$$
\tau=\frac{\left(x-x_{F O E}\right)}{u}=\frac{\left(y-y_{F O E}\right)}{v}
$$

which is equivalent to

$$
\tau=\frac{\sqrt{\left(x-x_{F O E}\right)^{2}+\left(y-y_{F O E}\right)^{2}}}{\sqrt{u^{2}+v^{2}}},
$$

where $x$ and $y$ are the coordinates of the center of the region under consideration, $u$ and $v$ are the components of the optical flow vector in such a region, and $x_{F O E}$ and $y_{F O E}$ are the coordinates of the FOE in the image.

Once the time to contact correspondent to each region in the image is available, the time to contact associated to each object is obtained by averaging the times to contact correspondent to the regions associated to that object. Then, the least time to contact corresponding to each column of the matrix of $24 \times 32$ optical flow vectors is obtained, thus filling a vector of 32 positions, whose elements are the values of the least time to contact corresponding to each vertical 10 pixels-wide stripe in the image frame. This is equivalent to define a set of 32 visual sensors, each one measuring the time to contact in an angle of $48.8 / 32=1.525$ degrees in the visual field of the robot. Such a vector, which is graphically depicted in Figure 3 , is the sensorial information the sensing system delivers to the control system in order to allow it to define the new heading angle to be imposed to the robot to avoid the obstacles in its visual field. Equal values of time to contact in contiguous positions of such a vector represent the distance from the robot to a single object in its field of vision. By its turn, distinct values of time to contact correspond to distinct objects. This way, in part c of Figure 3 it is possible to identify two different objects in the visual field of the robot, whose depths are characterized for the two clearly distinct values of time to contact.

The vector of times to contact and the magnitude of the optical flow vector in the regions associated to an object can provide rich information to a control system. In the sensing system here proposed, objects for which the average magnitude of the optical flow vectors exceed a threshold of 1.2 are understood as objects too close to the robot, thus demanding a more drastic evasive maneuver.

Important information is also provided by the average value and the standard deviation of the components of the vector of times to contact. When the standard deviation is too low, it is very likely that a single object spanning over the entire field

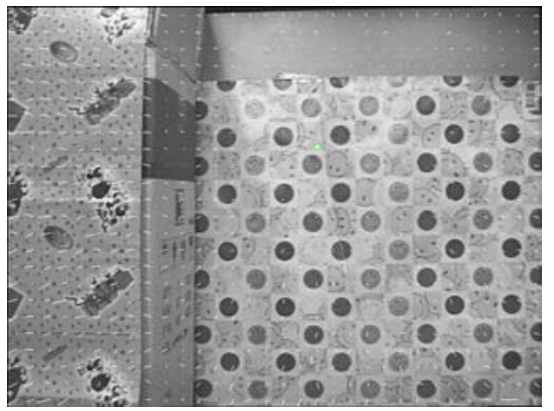

(a)

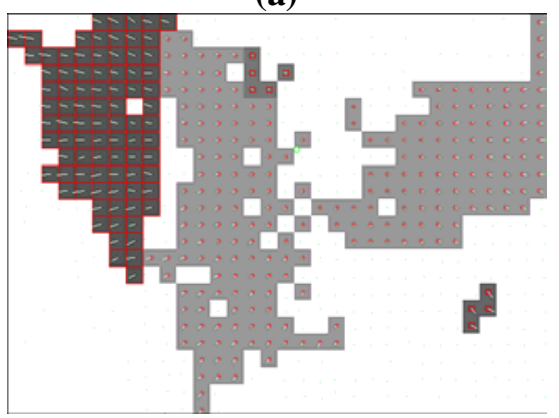

(b)

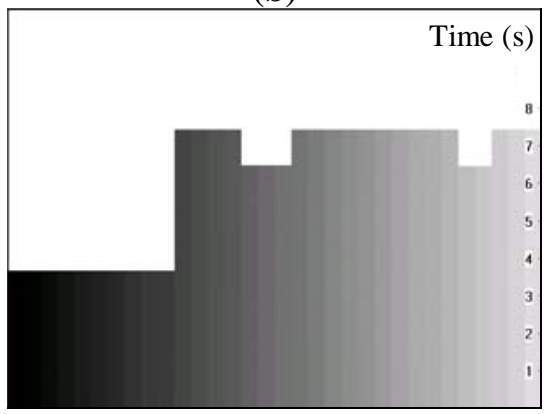

(c)

Figure 3: An example of the use of the optical flow-based image segmentation algorithm. In (a) it is shown the first of a set of two image frames acquired at the rate of 30 frames per second. In (b) it is shown the result of the image segmentation process (the white regions are those defined as not pertaining to an object in the scene, and the other regions are the objects detected - the darker the region is, the closer the object is). Finally, (c) depicts the vector of times to contact corresponding to such image frame.

of vision of the robot is in front of it. If the average time-tocontact value is also low (below $2.5 \mathrm{~s}$ ), this means that the object in front of the robot is very close to it. In both cases, to stop the robot is the safest reaction.

Finally, it is important to stress that the time spent to make all these analysis over the vector of times to contact is very small, because just a few elements are involved in the calculations. An analysis of how to use all the information the vector of times to contact provides, and how much time is 
consumed to do it, is presented in Section 5, in connection to the discussion of the results of an experiment run.

\section{CHECKING THE PERFORMANCE OF THE PROPOSED SENSING SYSTEM}

In this section a state-machine is designed in order to allow checking how the sensing system described in the above sections performs. The robot is programmed to wander around the lab, just avoiding colliding to any obstacle. It reacts to the presence of an obstacle by changing its heading angle from its current value (zero degrees, since the robot is just going ahead) to a new value, which is determined by the sensorial information embedded in the vector of times to contact delivered by the sensing system here proposed.

Three distinct situations are considered in the implementation of such a state-machine, which are labeled imminent collision ahead, side obstacle and normal situation. Imminent collision ahead is characterized either when the average value of the components of the vector of times to contact is below $2.5 \mathrm{~s}$ with a standard deviation close to zero (meaning that a wide object is very close to the robot), or when an object whose average magnitude of the optical flow vectors is greater than the threshold adopted is detected in the middle of an image frame (the elements 9 to 24 of the vector of times to contact). In such cases, the action the robot takes is to go back about $10 \mathrm{~cm}$, to rotate 180 degrees and to move ahead again. Those two situations are illustrated in Figure 4, were a wall is detected in front of the robot, and Figure 5, where the robot gets close to a waving hand, respectively.

Whenever the object whose average magnitude of the optical flow vectors is greater than the threshold value is in the right side (columns 25 to 32 ) or in the left side (columns 1 to 8 ) of the visual field of the robot, the situation labeled side obstacle is characterized. In such cases, the maneuver adopted is to rotate 15 degrees in the opposite direction and to resume moving ahead. Figure 6 illustrates a case of a side obstacle at the right side of the image frame. If two objects are detected, one in each side of the image frame, however, the system characterizes the situation as imminent collision ahead.

The normal situation is characterized when neither objects with high average optical flow magnitude nor objects spanning over all the visual field of the robot are detected in the image frame. This means that an abrupt maneuver is not necessary. When this is the case, a new small heading angle is determined, the robot turns around its own axis according to such angle and after continues moving ahead. This situation is illustrated in Figure 7, which shows two images collected by the camera onboard the robot. A certain time has elapsed and six deviations have been performed, after getting the two images shown in the figure. From the two images one can

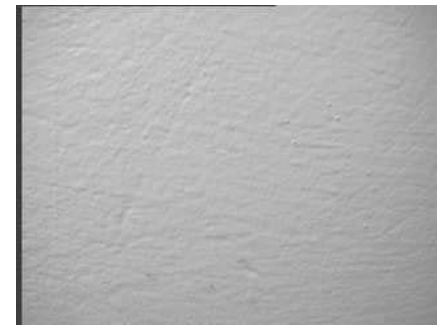

(a)

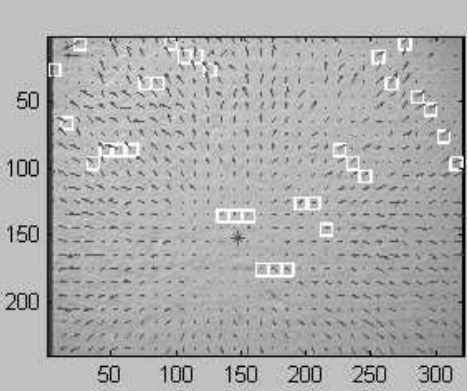

(b)

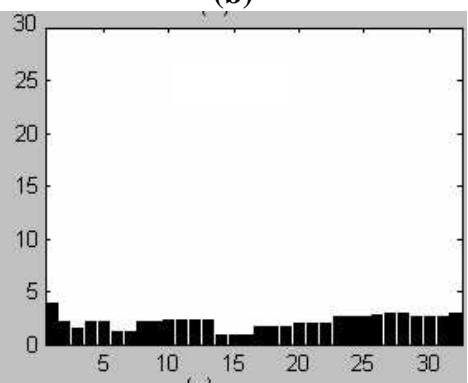

(c)

Figure 4: Exemplifying the detection of a wall. Part (a) shows the wall, part (b) shows the optical flow vectors calculated, and part (c) shows the corresponding vector of times to collision.

notice that the robot has deviated a little to the right, firstly because of the chair leg and after because of the table leg, both appearing in the first image and not in the second one.

In this case, the new heading angle to be imposed to the robot is determined according to the proposal discussed in (Sarcinelli-Filho et. al., 2002a). Like there, each element of the vector of times to contact is considered as similar information coming from distinct sensors. The state-machine associates to each sensor an angle, in order to make the robot to deviate of an obstacle detected in such a direction. The angle correspondent to a right turn, for the $i$-th component of the vector of times to contact, is given by

$$
\theta_{r i}=-\frac{\alpha_{r i}}{\tau_{i}+1}, \text { for } i=0,1, \ldots, 31,
$$

where $\alpha_{r i}=k i, k$ being a constant. Thus, the more right the 


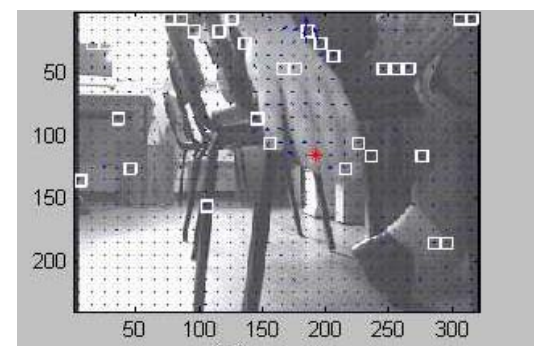

(a)

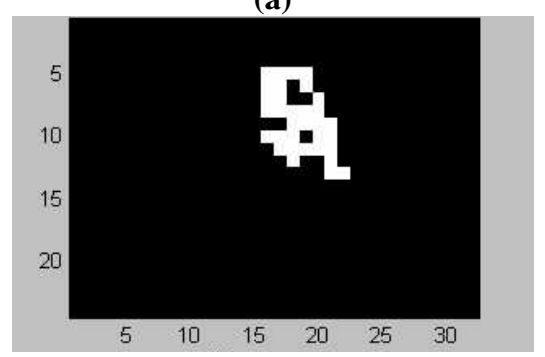

(b)

Figure 5: Illustrating the detection of a waving human hand in the middle of the image. The real hand is in (a), while (b) shows the regions whose average optical flow magnitude is higher than the threshold adopted.

column $i$ is, the greater the angle $\theta_{r i}$ is. In a similar way, the angle correspondent to a left turn, for the $i$-th component of the vector of times to contact, is given by

$$
\theta_{l i}=\frac{\alpha_{l i}}{\tau_{i}+1}, \text { for } i=0,1, \ldots, 31
$$

where $\alpha_{l i}=k(31-i), \quad k$ being a constant. In opposition to the right turn angles, the more right the column $i$ is, the smaller the angle $\theta_{l i}$ is. In the experiments, the value adopted for the constant $k$ was 3 .

The variances associated to such angles is given by

$$
\sigma_{i}^{2}=\tau_{i}, \text { for } i=0,1, \ldots, 31
$$

and represents an indication of the risk of a collision in the direction corresponding to each sensor, or, in other words, of the relevance of the obstacle in each column of the field of vision of the robot in terms of the robot safety.

The angles $\theta_{r i}$ and $\theta_{l i}$, and the respective variances, are inputted to two distinct decentralized Kalman filter (Brown and Hwang, 1997), which generates the overall left turn and right turn angles $\theta_{l}$ and $\theta_{r}$, respectively (in the range from - 15 degrees to +15 degrees), which are the two candidates to be the new heading angle of the robot. The new value for the robot heading angle is then defined as $\theta=\min \left(\theta_{l}, \theta_{r}\right)$.

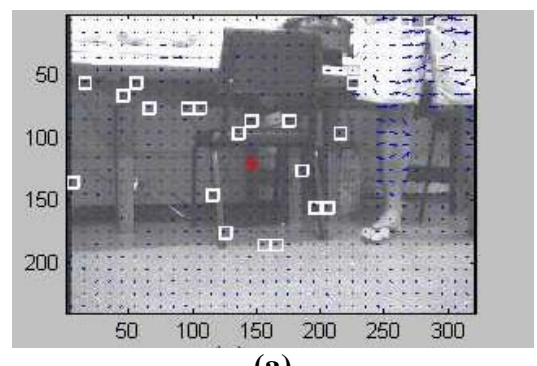

(a)

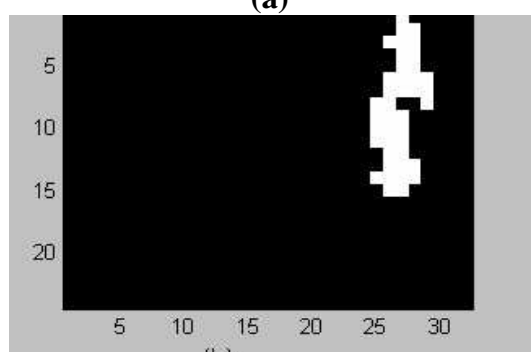

(b)

Figure 6: Detecting an object at the right side of the image. The object - a human leg - is in part (a), while part (b) shows the regions whose average optical flow magnitude is higher than the threshold adopted.

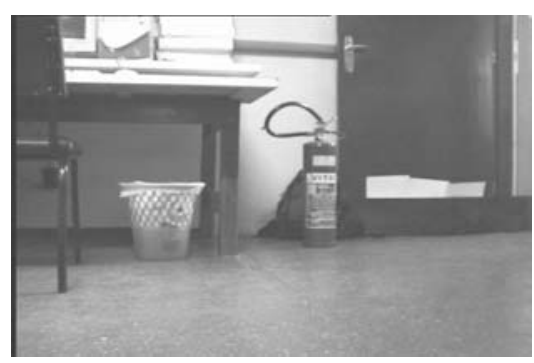

(a)

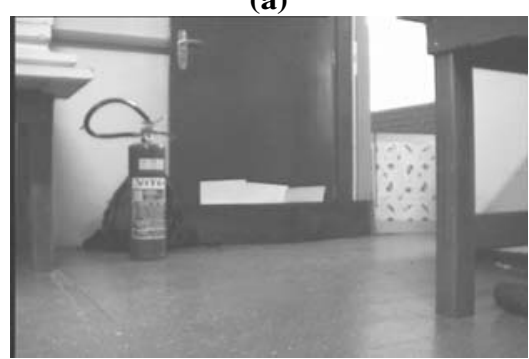

(b)

Figure 7: Illustrating the situation in which the robot performs smooth deviations (called normal situation). The images in parts (a) and (b) are two non-sequential images collected by the camera onboard the robot during its navigation. 


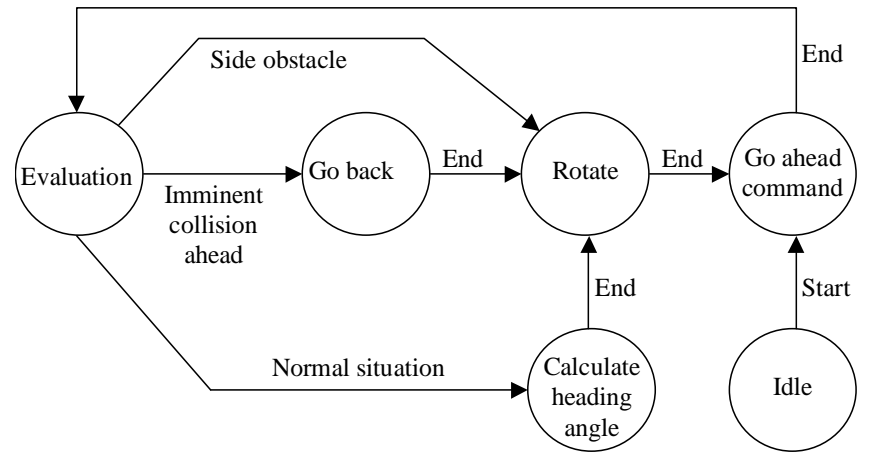

Figure 8: The state-machine corresponding to the control system implemented.

The three maneuvers above described represent the state changes of interest in the state machine implemented, which is depicted in Figure 8. There, all the optical flow calculation and image segmentation, up to generating the vector of times to contact and checking the average optical flow magnitudes for the regions pertaining to the objects detected, are performed when the robot gets into the state Evaluation. The other circles in Figure 8 correspond to the three commands above mentioned (to go back $10 \mathrm{~cm}$, to rotate and to move ahead), whose composition implements the above described composite maneuvers.

The state Idle in Figure 8 characterizes the robot state before being started. After the programmer starts the robot, it starts moving ahead, thus entering the state Evaluation. All the calculations necessary to determine the situation the robot is facing (imminent collision ahead, side obstacle or normal situation) are then performed, and, according to the situation, the suitable maneuver is implemented. In the normal situation case, the next state is to calculate the new heading angle (the current heading angle is always zero degrees).

It is also important to mention that the links included in Figure 8 are the only valid possibilities for our purposes. Any other link is not meaningful in the context here considered, and then is not included in the state-machine of Figure 8.

It should be emphasized, in such a context, that the command execution is strictly sequential, as defined by the branches End in Figure 8. Only after the system finishes the calculation of the new heading angle the corresponding turn around can start, as well as only after finishing turning around the robot can start moving ahead again. In particular, the action to move ahead demands that the robot linear speed starts in zero and gets its final value (in this work $100 \mathrm{~mm} / \mathrm{s}$ ), thus demanding a certain time to be accomplished. Hence, the image frames used in the optical flow calculation are always acquired when the robot is performing pure translation, thus guaranteeing that the FOE is always in the image, a necessary condition to calculate any time to contact.

\section{EXPERIMENTAL RESULTS}

In order to check the performance of the sensing subsystem discussed in Sections 2 and 3, under the coordination of the state-machine discussed in Section 4, an experiment is now presented and discussed. The robot is programmed to wander around in the lab, avoiding all the chair legs, table legs, people legs, walls, etc., it detects. Figure 9 shows the path the robot followed during its navigation. The layout of the lab has been over imposed to the figure in order to allow analyzing the maneuvers the robot performed.

In such a figure one can see that the robot stopped and rotated $180^{\circ}$ when facing either a wall or a static person (points $\mathrm{A}, \mathrm{B}$, and $\mathrm{C}$ ). All the remaining time the maneuvers executed corresponds to the normal situation. An analysis of all the actions the robot took shows that it was effectively able to avoid the obstacles that appeared in its way, as expected, using only the optical flow-based sensorial information, packed on the vector of times to collision discussed in Section 3. Other examples have also shown that using optical flow it is possible to detect the movement of people inside the lab, which suggests the possibility of using such sensing system to follow a person, for example.

In addition to Figure 9, Table II shows the times corresponding to the definition of the new heading angle and to the execution of the evasive maneuvers defined some times the robot got into the state Evaluation. There, a negative angle means a turn to the right, while a positive angle means a turn to the left. Also, the movements the robot executes are the composition of going back $10 \mathrm{~cm}$, rotating $180^{\circ}$ and going ahead (lines 5,8 and 11 of the table) and the composition of rotating much less than $180^{\circ}$ and going ahead (the other lines of the table), as discussed in connection to Figure 8. By to go ahead, one should understand that the robot goes ahead, according to the new heading angle established, and its linear speed increases up to $100 \mathrm{~mm} / \mathrm{s}$, which consumes the time specified in Table II (about $1 \mathrm{~s}$ ).

As one can see, the time corresponding to the acquisition of two image frames, the calculation of the optical flow vectors plus the calculation of the new heading angle is compatible with the robot dynamics, thus showing that the use of optical flow for this kind of sensing is suitable, which is one of the contributions of this paper.

Another feature to be mentioned is that the time corresponding to each cycle varies from about 1.8 seconds to about 4.8 seconds (see Table II), thus characterizing an asynchronous system. Therefore, it is not possible to define an image ac- 


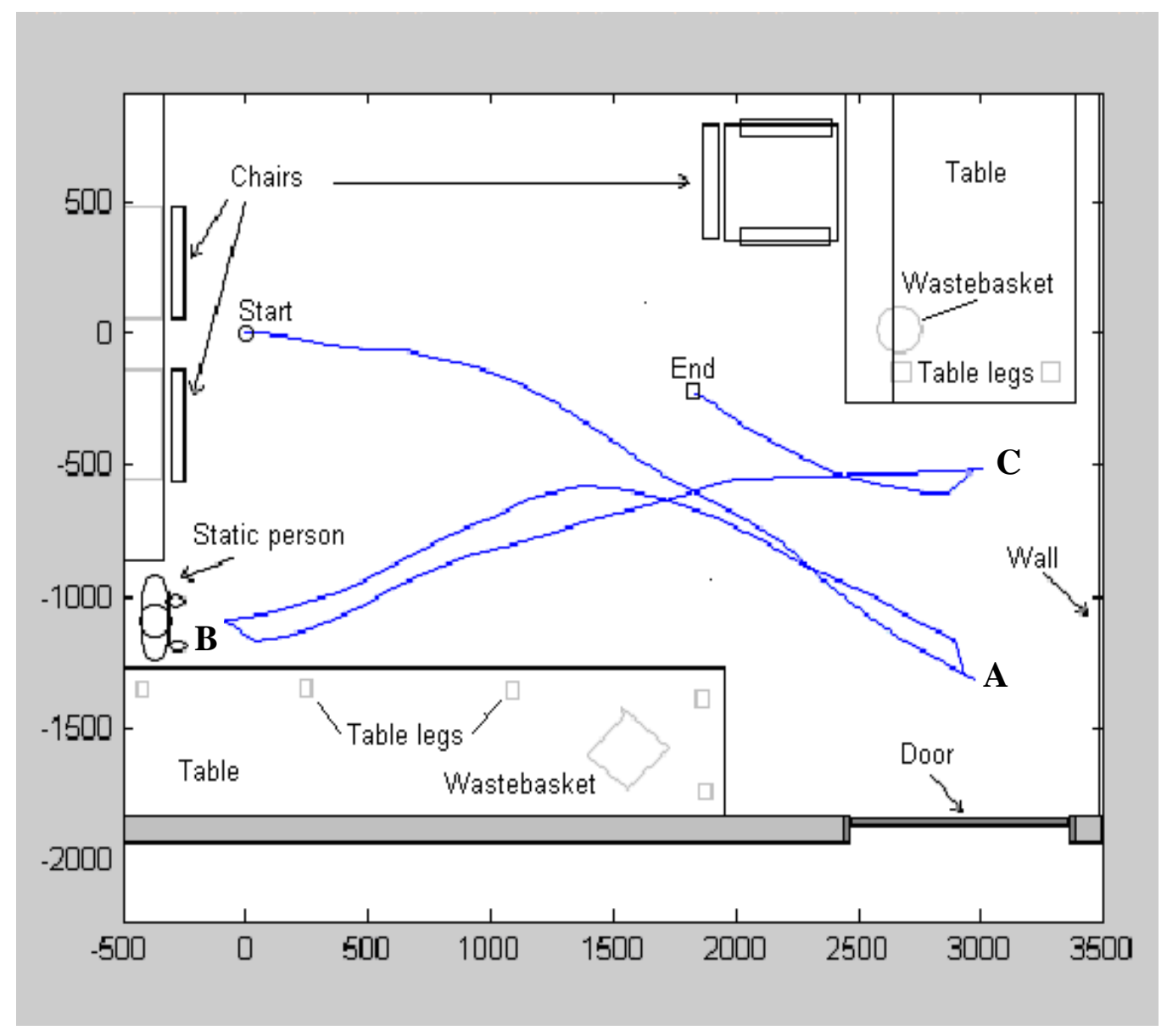

Figure 9: The path followed by the robot while wandering (distances are in $\mathrm{mm}$ ).

quisition rate. In addition, each image acquisition, included in the state Evaluation shown in Figure 8, corresponds to the acquisition of two subsequent image frames, and the time to capture both frames is also not constant. It depends on the processes being executed in the onboard computer (a Windows ME computer).

Another important result, regarding the time table corresponding to the experiment, is summarized in Table III. It illustrates the time spent to acquire the two image frames, to calculate the optical flow vectors, to generate the vector of times to collision, and to compare the average optical flow magnitudes correspondent to the different objects detected in the scene to the threshold adopted, for the same time instants registered in Table II. From such table, one can see that the most time consuming step of processing involved in the sensing subsystem is just the acquisition of the two image frames used to determine the optical flow vectors. Thus, the restriction on the number of image frames used by the algorithms for optical flow calculation is absolutely necessary, if one wants to reduce the time spent to get the sensorial infor- mation, as well as any attempt to improve the system reactivity should address a drastic change in the image acquisition procedure.

Other meaningful feature emerging from the analysis of Table II and Table III is that the whole time spent in the sensing subsystem processing is different in the two tables, which is due to the fact that such measures are performed in two distinct threads of the software corresponding to the system implementation, so that the measure presented in Table II includes the necessary passage of parameters from one to the other thread.

\section{CONCLUSION}

An optical flow-based sensing system is here proposed, which delivers to the robot control system a vector of times to collision, which resembles a map of depths. Each element in this vector represents the time within which the robot will reach the closest object in a vertical stripe of 10 pixels in the image frame considered. This way, the robot is able to make 
Table 3: The time table corresponding to the computations involved in the sensing subsystem.

\begin{tabular}{|c|c|c|c|c|c|c|}
\hline $\begin{array}{c}\text { Image } \\
\text { Acquisition } \\
\text { (ms) }\end{array}$ & $\begin{array}{c}\text { Optical } \\
\text { Flow } \\
\text { Calculation } \\
(\mathrm{ms})\end{array}$ & $\begin{array}{c}\text { Image } \\
\text { Segmentation } \\
(\mathrm{ms})\end{array}$ & $\begin{array}{c}\text { Determination } \\
\text { of the FOE } \\
\text { Coordinates } \\
(\mathrm{ms})\end{array}$ & $\begin{array}{c}\text { Analysis of } \\
\text { Characteristics } \\
(\mathrm{ms})\end{array}$ & $\begin{array}{c}\text { Calculation of } \\
\text { the New } \\
\text { Heading } \\
\text { Angle (ms) }\end{array}$ & $\begin{array}{l}\text { Total } \\
(\mathrm{ms})\end{array}$ \\
\hline 99 & 44 & 6 & 2 & 2 & 8 & 161 \\
\hline 53 & 44 & 6 & 1 & 3 & 7 & 114 \\
\hline 62 & 44 & 6 & 1 & 2 & 8 & 123 \\
\hline 60 & 50 & 6 & 2 & 2 & - & 121 \\
\hline 72 & 43 & 6 & 2 & 2 & 7 & 132 \\
\hline 52 & 43 & 6 & 2 & 2 & 7 & 112 \\
\hline 81 & 44 & 6 & 1 & 2 & - & 134 \\
\hline 56 & 44 & 8 & 1 & 2 & 7 & 118 \\
\hline 75 & 44 & 6 & 1 & 2 & 7 & 135 \\
\hline 79 & 44 & 6 & 1 & 2 & - & 132 \\
\hline
\end{tabular}

Table 2: The time-table corresponding to the experimentation.

\begin{tabular}{|c|c|c|c|c|}
\hline $\begin{array}{c}\text { Time to } \\
\text { acquire } \\
\text { two } \\
\text { image } \\
\text { frames } \\
\text { and to } \\
\text { deter- } \\
\text { mine the } \\
\text { new } \\
\text { heading } \\
\text { angle } \\
\text { (ms) }\end{array}$ & $\begin{array}{c}\text { Time to } \\
\text { go back } \\
10 \mathrm{~cm} \\
\text { (ms) }\end{array}$ & $\begin{array}{c}\text { Time to } \\
\text { rotate } \\
\text { (ms) }\end{array}$ & $\begin{array}{c}\text { Rotation } \\
\text { angle } \\
\text { (de- } \\
\text { grees) }\end{array}$ & $\begin{array}{c}\text { Time to } \\
\text { go } \\
\text { ahead } \\
\text { (ms) }\end{array}$ \\
\hline 178 & - & 592 & -3 & 1004 \\
\hline 124 & - & 1071 & 10 & 1006 \\
\hline 131 & - & 733 & 7 & 1006 \\
\hline 130 & 1005 & 2590 & 180 & 1005 \\
\hline 141 & - & 504 & 6 & 1010 \\
\hline 115 & - & 1020 & 12 & 1005 \\
\hline 137 & 1003 & 2584 & 180 & 1006 \\
\hline 121 & - & 1018 & -10 & 1011 \\
\hline 138 & - & 721 & -4 & 1006 \\
\hline 135 & 1005 & 2474 & 180 & 1007 \\
\hline
\end{tabular}

a decision on how much it should vary its current heading angle in order to avoid the closest obstacles in its visual field.
The two main paradigms in the conception of such a sensing system were the full onboard calculation and the guarantee that the robot were able to react to any environmental change in real-time. Such aims were effectively accomplished, as showed in the experiment reported.

As a result of the analysis of the experiments run, it is possible to conclude that the use of optical flow to implement reactive strategies for mobile robot navigation is viable. Actually, in the implementation here proposed it is shown that the main problem is not the computation time associated to the optical flow, time to contact or heading angle calculation, but the time consumed during the image acquisition.

\section{ACKNOWLEDGEMENTS}

The authors thank CNPq - National Council of Scientific and Technological Development, a Brazilian governmental institution promoting the scientific and technological development, for granting this research.

They also thank CAPES (Brazil) and SECyT (Argentina) for funding a partnership between Universidade Federal do Espírito Santo, Brazil, and Universidad Nacional de San Juan, Argentina, in which this work is included. As part of this partnership, Mrs. Eliete M. O. Caldeira got a scholarship to spend three months in San Juan, Argentina, working in this project.

Dr. Mário Sarcinelli-Filho also thanks FACITEC, a Vitória city hall organism promoting scientific and technological development, for the additional financial support granted. 


\section{REFERENCES}

Adams, R. and Bischof, L., (1994). Seeded Region Growing. IEEE Transactions on Pattern Analysis and Machine Intelligence, vol. 16, pp. 641-647, June.

Ancona, N. and Poggio, T., (1995). Optical Flow from 1D Correlation: Application to a Simple Time-to-Crash Detector. International Journal of Computer Vision, vol. 14, pp. 131-146.

Baratoff, G., Toepfer, C. and Neumann, H., (2000). Combined Space-Variant Maps for Optical Flow Based Navigation. Biological Cybernetics (Special Issue on Navigation in Biological and Artificial Systems), vol. 83, pp. 199-209.

Batavia, P. H., Pomerleau, D. A. and Thorpe, C. E., (1998). Overtaking Vehicle Detection Using Implicit Optical Flow. Proceedings of the IEEE Conference on Intelligent Transportation Systems, pp. 729-734.

Barraquand, J., Langlois, B. and Latombe, J. C., (1992). Numerical Potential Field Techniques for Robot Path Planning. IEEE Transactions on Systems, Man, and Cybernetics, vol. 22, pp. 224-241.

Barron, J. L., Fleet, D. J. and Beauchemin, S. S., (1993). Performance of Optical Flow Techniques. International Journal of Computer Vision, vol. 12, number 1, pp. 4377.

Barron, J. L. and Liptay, L., (1997). Measuring 3D Plant Growth Using Optical Flow. BioImaging, vol. 5, pp. 8286.

Bergen, J. R., Burt, P. J. and Hanna, K., (1992). Dynamic Multiple-Motion Computation. Artificial Intelligence and Computer Vision, Elsevier, The Netherlands, pp. 147-156.

Bleau, A. and Leon, L. J., (2000). Watershed-Based Segmentation and Region Merging. Computer Vision and Image Understanding, vol. 77, pp. 317-370.

Borshukov, G. D., Bozdagi, G., Altunbasak, Y. and Tekalp, A. M., (1997). Motion Segmentation by Multi-Stage Affine Classification. IEEE Transactions on Image Processing, vol. 6, number 11, pp. 1591-1594, November.

Brooks, R. A., (1986). A Robust Layered Control System for a Mobile Robot. IEEE Journal of Robotics and Automation, vol. 2, pp. 14-23, March.

Brown, R. and Hwang, P., (1997). Introduction to Randon Signals and Applied Kalman Filtering, 3rd edition, John Wiley \& Sons, New York, USA.
Carelli, R., Soria, C., Nasisi, O. and Freire, E. (2002). Stable AGV Corridor Navigation with Fused Vision-Based Control Signals. Proceedings of the IEEE 2002 28th Annual Conference of the Industrial Electronics Society - IECON 02, Sevilla, Spain, vol. 3, pp. 2433-2438, November.

Camus, T., Coombs, D., Herman, M. and Hong, T. H. (1999). Real-time Single-Workstation Obstacle Avoidance Using Only Wide-Field Flow Divergence. Videre: Journal of Computer Vision Research, MIT Press Journals, vol. 1, pp. 30-57.

Coombs, D., Herman, M., Hong, T. and Nashman, M., (1998). Real-time Obstacle Avoidance Using Central Flow Divergence and Peripheral Flow. IEEE Transactions on Robotics and Automation, vol. 14, pp. 49-59, February.

Dev, A., Kröse, B. J. A. and Groen, F. C. A., (1997a). Navigation of a Mobile Robot on the Temporal Development of the Optic Flow. Proceedings of the 1997 IEEE/RSJ/GI International Conference on Intelligent Robots and Systems (IROS'97), Grenoble, France, pp. 558-563, September.

Dev, A., Kröse, B. J. A. and Groen, F. C. A., (1997b). Confidence Measures for Image Motion Estimation. Proceedings of the 1997 RWC Symposium, Japan, pp. 199206.

Farah, M. J., (2000). The Cognitive Neuroscience of Vision - Fundamentals of Cognitive Neuroscience, Blackwell Publishers Inc., USA.

Giachetti, A., Campani, M. and Torre, V., (1998). The Use of Optical Flow for Road Navigation. IEEE Transactions on Robotics and Automation, vol. 14, pp. 34-48, February.

Grossmann, E. and Santos-Victor, J. A., (1997). Performance Evaluation of Optical Flow Estimators: Assessment of a New Affine Method. Robotics and Autonomous Systems, Elsevier, The Netherlands, vol. 21, pp. 69-82.

Horn, B. K. P. and Schunck, B. G. (1981). Determining Optical Flow. Artificial Intelligence, vol. 17, pp. 185-203.

Lai, S. H. and Vemuri, B. C., (1998). Robust and Efficient Computation of Optical Flow. International Journal of Computer Vision, vol. 29, pp. 87-105.

Lorigo, L. M., Brooks, R. A., Eric, W. and Grimson, L., (1997). Visually-Guided Obstacle Avoidance in Unstructured Environments. Proceedings of the 1997 IEEE/RSJ/GI International Conference on Intelligent Robots and Systems (IROS'97), Grenoble, France, pp. 373-379, September. 
Lucas, B. and Kanade, T., (1981). An Iterative Image Registration Technique with an Application to Stereo Vision. Proceedings of DARPA IU Workshop, pp. 121-130.

Nesi, P., Del Bimbo, A. and Ben-Tzvi, D. (1995). A Robust Algorithm for Optical Flow Estimation. Journal on Computer Vision, Graphics and Image Processing: Image Understanding, Academic-Press, vol. 61, number 2, pp. 59-68.

Ramel, G. J. L., (2000). The Incredible World of Mammals -The Sensory World of Mammals - Sight and Vision Sight and the Mammal Eye. The Earth Life Web, available in http://www.earthlife.net/mammals/vision.html, downloaded in April.

Santos-Victor, J. A., Sandini, G., Curotto, F. and Garibaldi, S., (1995). Divergent Stereo in Autonomous Navigation: From Bees to Robots. International Journal of Computer Vision, vol. 14, pp. 159-177, March.

Sarcinelli-Filho, M., Schneebeli, H. J. A., Caldeira, E. M. O. and Soria, C. M. (2002a). Optical Flow-Based Obstacle Detection and Avoidance in Mobile Robot Navigation. Proceedings of the $10^{\text {th }}$ IEEE Mediterranean Conference on Control and Automation - MED2002, in CD, Lisbon, Portugal, July.

Sarcinelli-Filho, M., Schneebeli, H. J. A., Caldeira, E. M. O., (2002b). Using Optical Flow to Control Mobile Robot Navigation. Proceedings of the $15^{\text {th }}$ World Congress on Automatic Control, Barcelona, Spain, July.

Stöffler, N. O., Burkert, T. and Färber, G., (2000). Real-Time Obstacle Avoidance Using an MPEG-Processor-Based Optic Flow Sensor. Proceedings of the 15th International Conference on Pattern Recognition, vol. 4, pp. 161-166, Barcelona, Spain, September.

Tekalp, A. M., (1995). Digital Video Processing, PrenticeHall, Englewood Cliffs, NJ, USA.

Wallach, D. S., Kunapalli, S. and Cohen, M. F., (1994). Accelerated MPEG Compression of Dynamic Polygonal Scenes," Computer Graphics (Proceedings of the ACM SIGGRAPH 1994), Orlando, FL, USA, pp. 193-196.

Wang, J. Y. A. and Adelson, E. H., (1994). Representing Moving Images with Layers. IEEE Transactions on Image Processing, vol. 3, pp. 625-638, September. 